\title{
Recombination of three ultracold fermionic atoms
}

\author{
H. Suno ${ }^{1}$, B.D. Esry ${ }^{1}$, and Chris H. Greene ${ }^{2}$ \\ ${ }^{1}$ Department of Physics, Kansas State University, Manhattan, Kansas 66506 and \\ ${ }^{2}$ Department of Physics and JILA, University of Colorado, Boulder, Colorado 80309
}

(Dated: October 29, 2018)

\begin{abstract}
Three-body recombination of identical, spin-polarized fermionic atoms in the ultracold limit is investigated. The mechanisms for recombination are described in terms of the "scattering volume" $V_{p}$ in the framework of the adiabatic hyperspherical representation. We have calculated numerically the recombination rate $K_{3}$ as a function of $V_{p}$ and have found that $K_{3}$ scales as $\left|V_{p}\right|^{8 / 3}$ for small $\left|V_{p}\right|$. A comparison with experimental data is also presented.

PACS numbers: 34.50.-s,34.10.+x,03.75.Fi
\end{abstract}

Recently, the quantum degenerate regime was attained in ultracold gases of fermionic atoms such as ${ }^{40} \mathrm{~K}[1]$ and ${ }^{6} \mathrm{Li}$ [2, 3]. Part of the motivation for these experiments is to observe a pairing of fermions, leading to a superfluid state. One important factor limiting the achievable density in these degenerate Fermi gases (DFG's) of trapped atoms is the loss of atoms through three-body recombination. Such losses occur when three atoms scatter to form a molecular bound state and a third atom $\mathrm{K}+\mathrm{K}+\mathrm{K} \rightarrow \mathrm{K}_{2}+\mathrm{K}$, for instance. The kinetic energy of the final state particles causes them to escape from the trapping potential.

While ultracold three-body recombination of identical, spin-polarized bosons has been theoretically studied because of its importance for Bose-Einstein condensates, recombination of identical fermions has not yet been con-

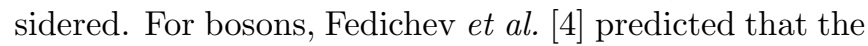
recombination rate $K_{3}$ grows with the two-body $s$-wave scattering length $a_{s}$, namely $K_{3} \propto a_{s}^{4}$, for $a_{s}>0$. This scaling was later confirmed by Nielsen and Macek [5] who also pointed out that it should hold for negative $a_{s}$. The $a_{s}^{4}$ scaling law for both signs of $a_{s}$ was indeed obtained by Esry et al. [6], Bedaque et al. [7], and Braaten and Hammer [8].

In the case of fermions, however, the Pauli exclusion principle prohibits $s$-wave scattering of atoms in identical spin states, thus leaving only $p$-wave collisions. The relevant low-energy scattering parameter in this case is the two-body $p$-wave "scattering volume" defined as

$$
V_{p}=-\lim _{k \rightarrow 0} \frac{\tan \delta_{p}(k)}{k^{3}}
$$

where $\delta_{p}(k)$ is the $p$-wave scattering phase shift and $k$ is the wave number. The scattering volume $V_{p}$ is related to the $p$-wave scattering length $a_{p}$ (see, for instance, Ref. [9]) by $V_{p}=a_{p}^{3}$. We choose $V_{p}$, rather than $a_{p}$, as the parameter to characterize the three-body recombination of fermions since an artificial nonanalyticity is introduced into $a_{p}$ when taking the cube root of the quantity in the right-hand side of Eq. (1).

Even though recombination of identical fermions is suppressed at ultracold temperatures by the Pauli princi- ple, it does not vanish. In fact, it has been shown that the rate is proportional to $E^{2}$ at low collision energies [12]. While this rate remains negligible under typical experimental conditions, it can become substantial near a Feshbach resonance. The $E^{2}$ threshold law no longer applies, and the recombination rate tends to the limit imposed by unitarity - often comparable to or larger than the rates for boson systems. Feshbach resonances are, of course, extremely useful tools for the experimentalist, so understanding the behavior near such a resonance is crucial. So far, such resonances have been observed, for example, in systems of ${ }^{40} \mathrm{~K} 10$ and ${ }^{6} \mathrm{Li}$ [2]. This resonant tuning was also observed for other alkali species in BEC experiments 11 .

This Letter treats the three-body recombination of identical, spin-polarized fermions in the ultracold limit. We examine the recombination rate $K_{3}$ as a function of the scattering volume $V_{p}$ by numerically solving the three-body scattering problem. First of all, since the atoms are spin polarized and thus in a completely symmetric spin state, the spatial wave function must be completely antisymmetric in order to satisfy the Pauli principle. This fact, combined with the generalization of Wigner's threshold law to $K_{3}$, shows that the $J^{\Pi}=1^{+}$ symmetry dominates at threshold, where $J$ is the total orbital angular momentum and $\Pi$ is the parity of the system. It follows that the recombination rate depends on the collision energy $E$ as $E^{2}$ near threshold [12]. (The same analysis applied to the boson case yields the familiar result that the recombination rate is constant at threshold due to the $0^{+}$symmetry.) Therefore, we consider only the $1^{+}$case. Dimensional analysis, together with this $E^{2}$ law, suggests a $\left|V_{p}\right|^{8 / 3}$ scaling of the recombination rate $K_{3}$ as opposed to the $a_{s}^{4}$ scaling for bosons.

The interaction potential used is a sum of triplet twobody potentials, i.e. $V=v\left(r_{12}\right)+v\left(r_{23}\right)+v\left(r_{31}\right)$. This choice is appropriate for fully spin-polarized atoms that collide in a quartet electronic state. For simplicity, we model the two-body potential as either

$$
v\left(r_{i j}\right)=D \operatorname{sech}^{2}\left(\frac{r_{i j}}{r_{0}}\right)
$$


or

$$
v\left(r_{i j}\right)=\frac{D}{1+\left(\frac{r_{i j}}{r_{0}}\right)^{6}} .
$$

The former potential has proven convenient in recombination calculations while the latter has a more physical van der Waal's tail with $C_{6}=r_{0}^{6} D$. Ideally, the results will not depend on the particular potential used in the ultracold limit. The parameter $r_{0}$ controls the range of the potential. The coefficient $D$, representing the potential depth, is treated as an adjustable parameter that permits us to control the scattering volume $V_{p}$, thus mimicking the tuning ability of an external magnetic field. This coefficient also economizes the calculations substantially since it allows us to reduce the number of two-body bound states without sacrificing the ultracold physics.

The two-body $p$-wave scattering volume $V_{p}$ behaves much like the $s$-wave scattering length as a function of the parameter $D$, displaying a tangent-like structure. As $D$ becomes more negative and the potential becomes more attractive, the scattering volume passes through a pole and changes sign each time the potential becomes deep enough to support one additional $p$-wave bound state. For simplicity, we consider the parameter range for which there exists only one two-body bound state.

The details of the theoretical methods we employ are more completely discussed in Ref. [13], so only a brief outline is given here. We use the adiabatic hyperspherical representation with modified Smith-Whitten hyperspherical coordinates. Simply put, hyperspherical coordinates transform the six relative Cartesian coordinates in the center of mass frame to a set with a single length coordinate, the hyperradius $R$, and five hyperangles. The hyperradius can thus be thought of as characterizing the overall size of the three-body system. These coordinates also allow us to easily impose the correct permutation symmetry on the wave functions. Solution of the adiabatic equation yields adiabatic hyperspherical potential curves and channel functions. The coupled hyperradial equations are then solved using an $R$-matrix propagation method.

The adiabatic hyperspherical representation reduces the collision of the three atoms to dynamics on a set of coupled hyperradial adiabatic potentials. These potentials bear a strong resemblance to standard molecular Born-Oppenheimer potentials (see Fig. 1) and can be interpreted in much the same way. The lowest potential curve in the figure, for instance, correlates to a bound molecule and a free atom far away. Because the atoms are identical fermions, the molecule has unit angular momentum and the potential has a centrifugal barrier. Further, because we consider only the $1^{+}$symmetry due to its dominance at threshold, the free atom must also be in a $p$-wave relative to the center of mass of the molecule. All of the other curves in Fig. 1 correlate to three free

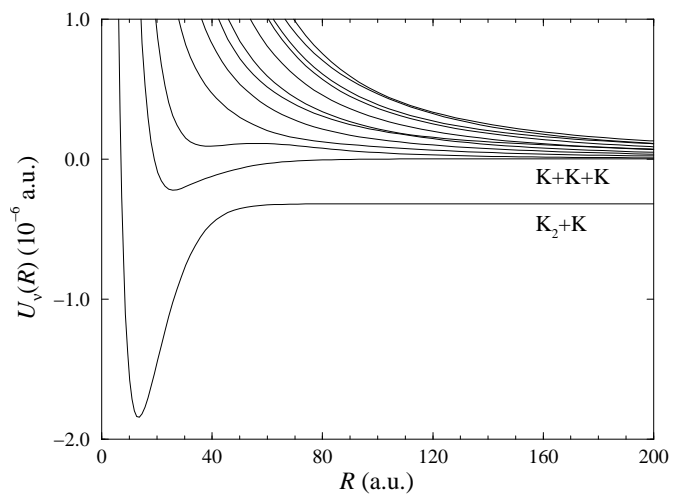

FIG. 1: The lowest 12 adiabatic hypersperical potential curves for three spin-polarized fermions with $V_{p}=-1.25 \times$ $10^{5}$ a.u. ${ }^{3}$ using the sech $^{2}$ potential with $r_{0}=15$ a.u.

atoms. In fact, there are an infinite number of potential curves associated with three-body continuum channels and approach the three-body breakup threshold $U=0$ asymptotically.

Three-body recombination can be seen as a transition from one three-body continuum channel to the recombination channel, driven by nonadiabatic coupling. Qualitatively, the adiabatic potentials shown in Fig. 11 display analogous behavior, as functions of $V_{p}$, as the adiabatic potentials for bosons show as functions of $a_{s}$ [6, 17] For both systems, the entrance channel goes from being strongly repulsive for positive $V_{p}$ and $a_{s}$ to having an attractive well behind a potential barrier for negative values. The entrance channel for the fermions is generally more repulsive, however, than the addition of a simple $J=1$ centrifugal potential term to the boson curves would give due to permutation symmetry considerations 12]. The recombination channels are also very similar, although it should be noted that there is no Efimov effect [14] for fermions in the limit $\left|V_{p}\right| \rightarrow \infty$. Efimov physics plays a key role in the interpretation of the ultracold recombination of bosons [6].

The primary difference between fermion and boson systems lies in the nonadiabatic coupling. While it is still similar for negative $V_{p}$ and $a_{s}$, for positive values the similarities end. Where the coupling strength for bosons shows a definite peak whose position increases linearly in $a_{s}$, the coupling strength for fermions remains peaked at small $R$ with a slowly decaying shoulder whose extent grows in proportion to $V_{p}^{1 / 3}$. For negative values of $V_{p}$, we thus expect that the fermion recombination rate can show resonant enhancement due to three-body shape resonances just as for bosons, but we do not expect an infinite series of such resonances since this was a consequence of the Efimov effect. We have not, however, seen evidence of such resonances as $V_{p} \rightarrow-\infty$ in our calculations.

To calculate the three-body recombination rate, we 
solve the hyperradial equations 13 for potentials like those shown in Fig. 1. The numerical hyperradial wave functions are matched to the appropriate Bessel functions based upon the known form of the long-range potentials [13]. The $S$-matrix is obtained and the generalized cross section for three-body recombination is calculated:

$$
\sigma=\frac{192(2 J+1) \pi^{2}}{k^{5}} \sum_{f, \lambda}\left|S_{f, \lambda}\right|^{2}=\frac{576 \pi^{2}}{k^{5}} \sum_{f, \lambda}\left|S_{f, \lambda}\right|^{2}
$$

Here, $k=\sqrt{2 \mu E / \hbar^{2}}$ is the hyperspherical wave number in the incident three-body continuum channel, and the indices $\lambda$ and $f$ label initial three-body continuum and final recombination channels, respectively. As it turns out, the numerical prefactor in Eq. (4) - which is determined by permutation symmetry - is the same as for three identical bosons [6] (recall that we are considering the $J^{\pi}=1^{+}$symmetry). The event rate constant per atomic triad is then defined simply as $K_{3}=\frac{\hbar k}{\mu} \sigma$. This quantity is related to the atom-loss rate constant $L_{3}$ by $L_{3}=3 K_{3} / 6$ [6].

Because the rate depends strongly on the collision energy in the ultracold regime, it must be thermally averaged in order to compare with experimental data. Following Ref. [16], we have derived the thermally averaged recombination rate constant to be

$$
\begin{aligned}
\left\langle K_{3}\right\rangle(T) & =\frac{\int K_{3}(E) E^{2} e^{-E / k_{B} T} d E}{\int E^{2} e^{-E / k_{B} T} d E} \\
& =\frac{2}{\left(k_{B} T\right)^{3}} \int K_{3}(E) E^{2} e^{-E / k_{B} T} d E .
\end{aligned}
$$

We show in Fig. 2 $\left\langle K_{3}\right\rangle$ as a function of $V_{p}$ for a temperature of $2 \mu \mathrm{K}$. Specifically, Fig. 2 2 shows $\left(K_{3} / r_{0}^{4}\right)^{3 / 8}$ versus $V_{p} / r_{0}^{3}$. We plot $K_{3}^{3 / 8}$ to best reveal whether the predicted $\left|V_{p}\right|^{8 / 3}$ scaling actually holds for the calculated rate; the factors of $r_{0}$ were included based on general arguments of length scale invariance of the Schrödinger equation. Because the two-body potentials in Eqs. (2)-(3) depend only on the combination $r_{i j} / r_{0}, r_{0}$ can be defined to be the new length scale for both the two-body and threebody systems. This new length scale implies multiplying energies and temperatures by $r_{0}^{2}$, lengths by $r_{0}^{-1}$, and the rate by $r_{0}^{-4}$. The energy parameter $D$ in our model which controls $V_{p}$ - is independent of the length scale, and so its scaled counterpart, $r_{0}^{2} D$, can be used to control $V_{p} / r_{0}^{3}$. Having calculated the rate as a function of $V_{p}$ and energy for a fixed $r_{0}$, we can thus obtain the rate for any other value of $r_{0}$ by means of the above scaling. The key is that at a fixed laboratory collision energy (or temperature), changing $r_{0}$ effectively changes the range of the two-body potential, and the new rate corresponds to the rate at the scaled energy $r_{0}^{2} E$. One has to be careful, of course, to track the simultaneous change in the scaled $V_{p}$.

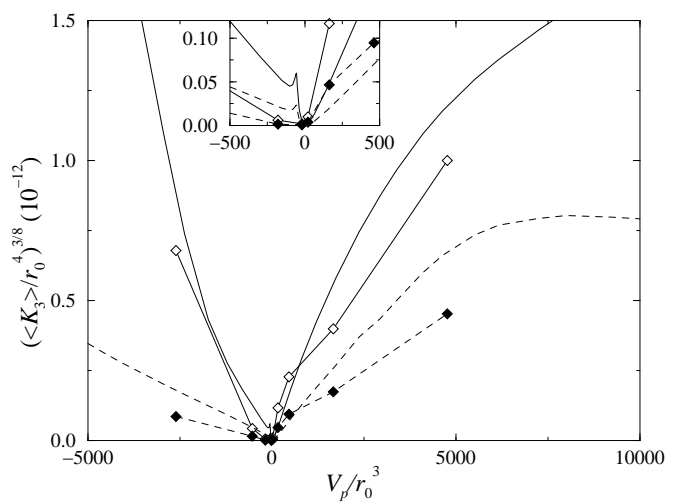

FIG. 2: The recombination rate $K_{3}$ as a function of $V_{p}$ for potentials from Eq. (2) (no symbols) and Eq. (3) (diamonds). The solid lines corresponds to the thermally averaged rate at $T=2 \mu \mathrm{K}$; and the dashed lines, to the energy-dependent rate at $E=2 \mu \mathrm{K}$. All curves were calculated with $r_{0}=15$ a.u. The inset focuses on the region with small $\left|V_{p}\right|$ showing a Stückelberg oscillation.

The solid lines in the figure show the thermally averaged rates $\left\langle K_{3}\right\rangle(T)$, while the dashed lines show the energy-dependent rates $K_{3}(E)$. The symbols for the $\operatorname{sech}^{2}$ potential, Eq. (2), are omitted for clarity since the rate was obtained on a relatively dense set of $V_{p}$ values. The diamonds denote the rates resulting from the second potential, Eq. (3). The rates from the two potentials which are qualitatively very different - show reasonable quantitative agreement, especially for the more experimentally relevant thermally averaged rate. The poorest agreement occurs at small $\left|V_{p}\right|$ where the rates are also small. If, however, the rate curve for either potential with a different value of $r_{0}$ and the same lab energy $E$ were plotted here, it would look systematically different, although qualitatively similar. This apparent dependence on the two-body potential would seem to argue against a "universal" curve as was found for bosons [5, 6, 7, 8. Based on the above length scaling arguments, though, it is clear that the scaled rate curves - at the same scaled energy - are what should be compared between different potentials and would thus come closest to a universal curve.

Figure 2 shows that the $\left|V_{p}\right|^{8 / 3}$ scaling does indeed hold quite well for $\left|V_{p}\right| / r_{0}^{3}$ less than roughly 2500 for $K_{3}(E)$, but for $\left\langle K_{3}\right\rangle(T)$ it only holds over the region $\left|V_{p}\right| / r_{0}^{3}$ below approximately 800 . Since this scaling is based on the threshold behavior of the rate, it is not surprising that it breaks down sooner for the thermally averaged rate given that the averaging procedure includes rates from higher energies. The fact that the scaling breaks down for $K_{3}(E)$ is an indication that the fixed collision energy is no longer in the threshold regime.

For large positive scattering volumes $V_{p}$, the collision energy eventually becomes large compared to the dimer binding energy, and $K_{3}$ increases more slowly than the 
$\left|V_{p}\right|^{8 / 3}$ scaling law. Recalling that for negative $V_{p}$ there is a barrier in the initial adiabatic hyperspherical potential, the breakdown in the scaling law comes when the barrier sinks below the collision energy at large negative $V_{p}$. The fact that the recombination rate increases more rapidly than the scaling law with increasing $\left|V_{p}\right|$ simply reflects the more ready tunneling through the barrier. The rate continues to increase until it reaches the limit imposed by unitarity, namely (in SI units)

$$
K_{3}^{\max }=\frac{\hbar}{\mu} \frac{576 \pi^{2}}{k^{4}}=\frac{\hbar^{5}}{m^{3}} \frac{144 \sqrt{3} \pi^{2}}{E^{2}} .
$$

This value is obtained from Eq. (4) by assuming that the recombination predominantly comes from the lowest continuum channel with unit probability. We expect that this limit will be reached when $\left|V_{p}\right|$ becomes large enough that the barrier in the three-body entrance channel is lower than the collision energy. For the conditions of Fig. 2, this value of $V_{p}$ is well off the scale of the plot.

Interestingly, the inset of Fig. 2 2 shows a small peak in the rate located at about $V_{p} / r_{0}^{3}=-60$, i.e. at $\mathrm{B}=200.8$ G. This peak is a constructive interference between two indistinguishable pathways - a Stückelberg oscillation. This peak disappears and reappears as a function of collision energy since the phase difference between the two pathways depends on the energy.

In Fig. 3, we compare our calculations with experimental measurements of the recombination rate of three ${ }^{40} \mathrm{~K}$ atoms all in the $|9 / 2,-7 / 2\rangle$ spin state near a $p$-wave two-body Feshbach resonance. The theoretical rates are calculated at $T=2.5 \mu \mathrm{K}$, while the experimental temperature varies between 2 and $3 \mu \mathrm{K}$. The figure shows the rates as functions of the magnetic field strength $B$. The Feshbach resonance lies at $B_{0}=198.5 \mathrm{G} ; V_{p}$ is positive for $B$ below the resonance and negative for $B$ above. Treating $r_{0}$ as a fitting parameter, we obtain the solid line shown in the figure. The agreement on the rise of the resonance $\left(V_{p}>0\right)$ is better than on the fall $\left(V_{p}<0\right)$. The disagreement away from the resonance is likely due to the breakdown of the experimental assumption of purely three-body loss in determining the rate coefficient. Near the resonance, however, our theoretical rates between one and two orders of magnitude lower. In fact, for all values of $r_{0}$, the theoretical values lie below the experimental ones. This discrepancy is not surprising given that we included only a single partial wave $\left(1^{+}\right)$in our calculation. While this symmetry controls the limiting behavior at threshold, it is not necessarily dominant near the resonant peak. In fact, limited explorations have demonstrated that other $J^{\pi}$ can be comparable to $1^{+}$at the resonance peak. The contributions from these higher partial waves accumulate quickly since thermal averaging, from Eq. (5), gives an extra factor of roughly $(J+2)$ !.

In summary, we have investigated ultracold three-body recombination of identical spin-polarized fermions and have numerically calculated the recombination rate as a

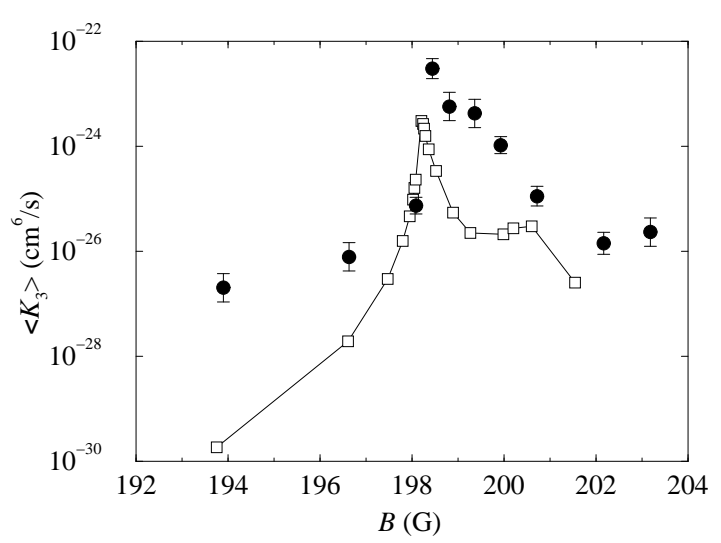

FIG. 3: The theoretical rate (solid line) and the rate measured by C. Regal et al. (filled circles) are plotted as functions of the magnetic field strength $B$.

function of the scattering volume. This is the first such study that we are aware of, and much remains to be understood about these fermion systems. Moreover, with some slight modifications to our numerical method, it is possible to treat systems with only two identical particles. Three-body recombination of such systems plays an important role in experiments on mixed-spin state Fermi gases and mixed Bose-Fermi gases.

This work was supported in part by the National Science Foundation and the Research Corporation. We thank C. Ticknor and J. Bohn for sharing their twobody theoretical scattering data. We also thank C. Regal and D. Jin for sharing their experimental recombination rates.

[1] B. DeMarco and D.S. Jin, Science 285, 1703 (1999).

[2] K.M. O'Hara et al., Phys. Rev. Lett. 85, 160407 (2000).

[3] A.G. Truscott et al., Science 291, 2750 (2001).

[4] P.O. Fedichev et al., Phys. Rev. Lett. 83, 2921 (1996).

[5] E. Nielsen and J.H. Macek, Phys. Rev. Lett. 83, 1566 (1999).

[6] B.D. Esry et al., Phys. Rev. Lett. 83, 1751 (1999). Note that the cross section formula in this reference, and the calculated rates, should be decreased by a factor of six.

[7] P.F. Bedaque et al., Phys. Rev. Lett. 85, 908 (2000).

[8] E. Braaten and H.-W. Hammer, Phys. Rev. Lett. 87, 908 (2001).

[9] J.L. Bohn, Phys. Rev. A 61, 053409 (2000).

[10] T. Loftus et al., Phys. Rev. Lett. 88, 173201 (2002).

[11] For example, S. Inouye et al., Nature (London) 392, 151 (1998).

[12] B.D. Esry et al., Phys. Rev. A 65 R010705 (2002).

[13] H. Suno et al., Phys. Rev. A 65, 042725 (2002).

[14] V.M. Efimov, Phys. Lett. B 33 (1970) 563.

[15] C.D. Lin, Phys. Rep. 257, 1 (1995).

[16] J.P. Burke, Jr., Ph.D. thesis, University of Colorado, 1999.

[17] B.D. Esry, et al. J. Phys. B 29, L51 (1996). 


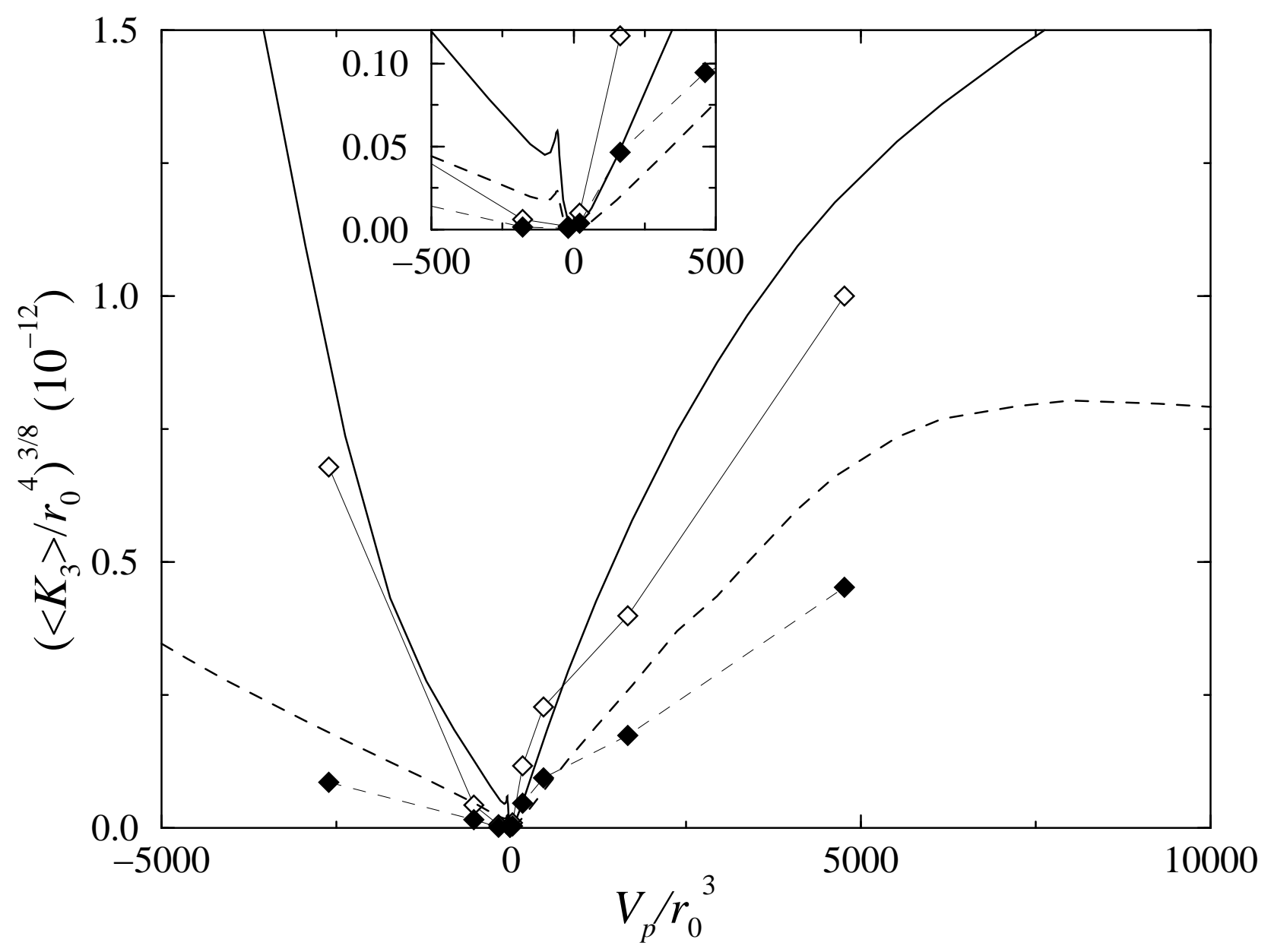

